\title{
Electronic properties of disclinated flexible membrane beyond the inextensional limit: Application to graphene
}

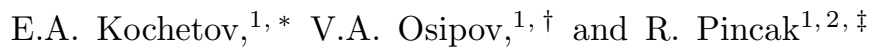 \\ ${ }^{1}$ Bogoliubov Laboratory of Theoretical Physics, Joint Institute for Nuclear Research, 141980 Dubna, Moscow region, Russia \\ ${ }^{2}$ Institute of Experimental Physics, Slovak Academy of Sciences, Watsonova 47,04353 Kosice, Slovak Republic
}

\begin{abstract}
Gauge-theory approach to describe Dirac fermions on a disclinated flexible membrane beyond the inextensional limit is formulated. The elastic membrane is considered as an embedding of $2 D$ surface into $R^{3}$. The disclination is incorporated through an $S O(2)$ gauge vortex located at the origin, which results in a metric with a conical singularity. A smoothing of the conical singularity is accounted for by replacing a disclinated rigid plane membrane with a hyperboloid of near-zero curvature pierced at the tip by the $S O(2)$ vortex. The embedding parameters are chosen to match the solution to the von Karman equations. A homogeneous part of that solution is shown to stabilize the theory. The modification of the Landau states and density of electronic states of the graphene membrane due to elasticity is discussed.
\end{abstract}

PACS numbers: 61.72.Lk; 73.22.Pr

Keywords:

\section{INTRODUCTION}

Is is now generally accepted that the low-lying electronic states in graphene can be accurately described by twodimensional massless Dirac fermions on a plane [1]. In experiment, multiform graphene structures were observed thus stimulating studies of Dirac fermions on curved graphene sheets (see, e.g., 2, 3]). This problem is markedly complicated when the curvature itself is generated by topological defects like disclinations. Indeed, a disclination is known in elasticity theory as a line defect which can be produced by "cut and glue" Volterra process, namely, by inserting or removing a wedge of material with the following gluing of the dihedral sides. This immediately generates additional large elastic strains inside the crystal. For flexible membranes, however, there is a chance to screen out the strain field by buckling into a cone. The problem thus reduces to coupling Dirac spinors to a topologically non-trivial curved background.

The topological lattice defects in graphene are pentagons that are equivalent to wedge disclinations. The first experimental observation of a pentagon at the apex of a cone was provided by An et al. in [4]. They used the scanning tunneling microscope to study the structure of a conical protuberance and found five bright spots at the apex of the nanocone. This was the first clear evidence that the pentagon is located at the apex. The bright spots indicate also that there is an enhanced charge density localized at each carbon atom in the pentagon, which implies an increase in the electronic density of states (DOS). This finding was confirmed by the numerical tight-binding calculations for nanocones with different number of pentagons at the apex [5]. Explicit manifestations of the topological effects in the electronic properties of disclinated rigid graphene surfaces have been discussed at length in the literature [6-15].

However, disclinations are the sources of long range elastic stresses which also modify the electronic states. The strain-induced effects in the electronic structure of graphene are now a topic of intense research in strain engineering to produce basic elements for all-graphene electronics. In particular, designed strains can generate electron beam collimation, confined states, quantum wires [16] as well as energy gaps and a zero-field quantum Hall effect [17] in graphene. To incorporate internal stresses due to topological defects one is supposed to go beyond the inextensional limit. Study of electronic properties of disclinated flexible membranes beyond the inextensional limit is thus of importance to address this new field of research.

According to Volterra process disclination can be considered as a conical singularity like strings in cosmology. The relevant background is the curved spacetime where all the curvature is concentrated at the apex of the cone. The metric in the cylindrical coordinates is written as

$$
d s^{2}=d t^{2}+d z^{2}+d r^{2}+\alpha^{2} r^{2} d \varphi^{2} .
$$

\footnotetext{
*Electronic address: kochetov@theor.jinr.ru

${ }^{\dagger}$ Electronic address: osipov@theor.jinr.ru

‡Electronic address: pincak@saske.sk
} 
Here the parameter $\alpha$ is related to the angular sector that is removed or inserted to form the defect. In this case, any attempt to build a closed loop around the disclination line will result in a closure failure. The deficit angle is equal to $2 \pi \alpha$ with $\alpha=1-\nu$ where $\nu$ is the Frank index, the basic topological characteristic of the disclination. The positive sign of $\nu$ corresponds to the removing of a sector. In this case the space has positive curvature. Correspondingly, for negative $\nu$ one has a cone of negative curvature. Eventually, the problem reduces to a Dirac equation in the curved spacetime. By the change $r \rightarrow r^{\alpha} / \alpha$ metric (11) can be brought into the form

$$
d s^{2}=d t^{2}+d z^{2}+r^{2(\alpha-1)}\left(d r^{2}+r^{2} d \varphi^{2}\right)
$$

which describes a featureless cosmic string located at the origin.

In spite of the elegant form of this approach, there is yet an important open question concerning the so-called core region of the defect. To the best of our knowledge, this problem was for the first time raised in cosmological models [18, 19] where long-range effects of cosmic string cores were studied. In geometric theory of defects, an influence of a disclination core on the localization of electrons and holes was investigated in 20]. In both cases, the tip of the conical singularity is replaced by a smooth cap while at large distances a typical cone with the deficit angle $2 \pi \alpha$ emerges. In cosmological models the curvature of an infinite string is confined within a cylinder of a small radius $a$ (the core radius) that possesses a direct physical meaning: the string has a characteristic core radius given by $a \approx 1 / M$, where $M$ is a mass scale at which the string is formed. Accordingly, the relevant $2 D$ piece of the metric can be taken in the form

$$
d s^{2}=P^{2}(r / a) d r^{2}+\alpha^{2} r^{2} d \varphi^{2},
$$

where the range of the angular coordinate is $\varphi \in[0,2 \pi)$ and $P(r / a)$ is a smooth monotonic function satisfying the conditions

$$
\lim _{r / a \rightarrow 0} P(r / a)=\nu, \quad P(r / a)=1, \quad r>a .
$$

For example, in [20] the so-called flower-pot model was considered when the curvature of the disclinated media is concentrated on a ring of radius $a$, which results in the formation of a "seam" on the cylinder.

This approach is of interest in the description of linear defects with a certain interior structure (finite thickness of a string). However, the situation is more subtle for a disclination on an elastic $2 D$ surface, e.g., on a graphene sheet. The specificity of elastic membranes lies in that they may change both their intrinsic and extrinsic geometries due to stretching and bending of the membranes, respectively. This occurs because a flexible membrane can relieve the internal strain by buckling out of the plane into a cone. In the inextensional limit of the infinite rigidity, the stretching energy of a membrane of the radius $R$ is proportional to the system size, $R^{2}$, whereas a buckled membrane has the energy proportional to $\log R$. In this case a creation of a disclination by using the "cut and glue" process results in a true cone. This corresponds to a point-like disclination defect. In reality, however, a membrane possesses finite elasticity, so that one needs to go beyond the inextensional-limit approximation.

As is known, the classical theory of elasticity introduces a characteristic velocity, but does not lead to a characteristic length. In defect theory the length scale is introduced phenomenologically through the core radius of the defect which appears as an external parameter. Accordingly, there is no room within the linear theory of elasticity for the description of the core region. In the rotationally symmetric case it represents a small disc with a certain radius $r_{0}$. Physically, the parameter $r_{0}$ comes into play through the boundary conditions imposed on the stress tensor to insure that the internal strain inside a membrane be kept finite to prevent it from falling apart. It is this quantity $r_{0}$ that sets a relevant short-range length scale in the present problem (similar to $a$ for cosmic strings).

In this paper, we attempt a variant of the self-consistent gauge-theory approach to account for both the smoothed apex and the topological characteristic of the disclination defect. To take into account finite elasticity, we go beyond the inextensional-limit approximation. In doing so, we invoke both the linear elasticity theory as well as a phenomenological approach to account for the elastic deformations. Dynamical variables in our theory are the embeddings of a $2 D$ elastic surface into $R^{3}$. Parameters of the embedding appear as the matter fields interacting with an external gauge potential that describes a disclination. Within the linear scheme the model recovers the von Karman equations for membranes with a disclination-induced source generated by the gauge field. We explicitly show that the elastic deformations emerge due to the defect. We compute the effective metric generated by the disclination. That metric is determined by the external gauge field and the dynamical embeddings. It contributes to a topologically nontrivial part of the spin connection that couples Dirac fermions to a $2 D$ disclinated elastic manifold.

The paper is organized as follows. Section II contains a brief account of a gauge-theory approach to describe fermions on a disclinated surface in the inextensional limit. To take into account elastic deformations, a dynamical theory beyond the inextensional-limit approximation is presented in Section III. Within that theory Dirac fermions couple to the underlying effective metric generated by the disclination defect in the presence of elasticity. In Section IV the derived approach is specified to study electronic properties of disclinated graphene. 


\section{INFINITE RIGIDITY}

In the present Section we show that a disclination on a $2 D$ manifold can be thought of as arising due to an explicit breaking of the local rotational symmetry. This breaking can be enforced by a topologically nontrivial external gauge field that carries the disclination "charge", $\nu$. To set the stage, we begin with a brief discussion of the case of an infinitely rigid membrane free to buckle into a cone in $R^{3}$. This corresponds to the inextensional limit to be discussed from the elasticity theory point of view in the next Section. Here we just show that the conical-type singularity generated by the vortex results in a topological nontrivial contribution to the spin connection for Dirac fermions. Our discussion essentially follows that of our earlier short Communication [21], except that it is extended now to include a disclination dipole as well.

\section{A. Disclination vs gauge field}

Let $x^{a}$ be a set of local coordinates on a Riemannian surface $\Sigma_{0}$. (Indices $a, b, c, \ldots=1,2$ are tangent to $\Sigma_{0}$, whereas $i, j, k, \ldots=1,2,3$ run over the basis of $\left.R^{3}\right)$. To describe this we find it convenient to introduce an embedding $\Sigma_{0} \rightarrow R^{3}$ that can be realized in terms of a $R^{3}$-valued function $R_{(0)}^{i}\left(x^{1}, x^{2}\right)$. As the point $\left(x^{1}, x^{2}\right)$ is varied, vector $\vec{R}_{(0)}$ sweeps the surface $\Sigma_{0}$. This is nothing but a familiar two-parametric representation of surfaces in $R^{3}$. In what follows the function $R_{(0)}^{i}\left(x^{1}, x^{2}\right)$ is chosen to specify an initial configuration $\Sigma_{0}$. Representation for the induced metrics follows immediately

$$
g_{a b}^{(0)} \equiv\left(g_{\Sigma_{0}}\right)_{a b}=\partial_{a} \vec{R}_{(0)} \cdot \partial_{b} \vec{R}_{(0)} .
$$

Equation (5) is invariant under global $S O(3)$ rotations of the vector $\vec{R}_{(0)}$. To incorporate disclinations one should promote this invariance to a local one. To this end, consider the $R_{(0)}^{3}$-bundle over $\Sigma_{0}$ with the structure group $S O(3)$. The $s o(3)$ valued one form $\left(\vec{W}_{a}^{(0)} \cdot \vec{L}\right) d x^{a}$ serves as a connection one-form in the $R_{(0)}^{3}$-bundle space over $\Sigma_{0}$, with $\vec{W}_{a}^{(0)}$ being the gauge potentials. Here $L^{i} \in s o(3)$ are the generators of the group. By replacing in (5) ordinary derivatives $\partial_{a} \vec{R}_{(0)}$ by the covariant ones $\nabla_{a} \vec{R}_{(0)}=\partial_{a} \vec{R}_{(0)}+\left[\vec{W}_{a}^{(0)}, \vec{R}_{(0)}\right]$, one arrives at the locally $S O(3)$ invariant representation for the induced metric,

$$
\begin{aligned}
g_{a b} & =g_{a b}^{(0)}\left(\vec{W}^{(0)}\right)=\nabla_{a} \vec{R}_{(0)} \cdot \nabla_{b} \vec{R}_{(0)}=\partial_{a} \vec{R}_{(0)} \cdot \partial_{b} \vec{R}_{(0)}+\partial_{a} \vec{R}_{(0)}\left[\vec{W}_{b}^{(0)}, \vec{R}_{(0)}\right]+\partial_{b} \vec{R}_{(0)}\left[\vec{W}_{a}^{(0)}, \vec{R}_{(0)}\right] \\
& +\left(\vec{W}_{a}^{(0)} \vec{W}_{b}^{(0)}\right) \vec{R}_{(0)}^{2}-\left(\vec{W}_{a}^{(0)} \vec{R}_{(0)}\right)\left(\vec{W}_{b}^{(0)} \vec{R}_{(0)}\right) .
\end{aligned}
$$

Topological disclinations can then be considered as arising due to explicit breaking of the local rotational symmetry by a fixed topologically nontrivial gauge potential that generates a new metric as follows from Eq. (6). ¿From now on a metric induced due to either the gauge field or elastic deformations will be denoted by $g_{a b}$ to reserve the symbol $g_{a b}^{(0)}$ for the metric tensor on the undisturbed surface, $\Sigma_{0}$.

In general case, a nonabelian gauge field $\vec{W}^{(0)}$ emerges to describe disclinated surface . However, throughout this paper we are primarily interested in the case $\Sigma_{0}=R^{2}$ so that only the $z$-component of the gauge field matters. To illustrate this, consider a disclination defect placed at the origin of a plane that can be bent but cannot be stretched. We have $\Sigma_{0}=R^{2}$, so that $\left(x^{1}=x, x^{2}=y\right) \in R^{2}$. In this case $W_{\mu}^{(0) i=1,2}=0$ and $W_{\mu}^{(0) i=3}=W_{\mu}^{(0)}$. A singular vortex-like potential

$$
W_{x}^{(0)}=\nu y / r^{2}, W_{y}^{(0)}=-\nu x / r^{2}, r=\sqrt{x^{2}+y^{2}} \neq 0
$$

is supposed to describe a topological disclination with a strength $\nu$ located at $\vec{r}=0$. This potential locally is a pure gauge,

$$
W_{a}^{(0)}(\vec{r})=-\nu \partial_{a} \tan ^{-1} \frac{y}{x}
$$

However, for any counter $C$ encircling the origin one has

$$
\oint_{C} W_{a}^{(0)} d x^{a}=-2 \pi \nu \neq 0
$$

Since the counter integral in Eq.(8) is a gauge invariant quantity, the field $W_{\mu}^{(0)}$ cannot be gauged away to zero due to the topological obstruction. This is why that field is referred to as a topologically non-trivial one. A physically 
observable quantity associated with that gauge field is a nonzero flux, $\Phi=-2 \pi \nu$, through an area bounded by the counter $C$. It does not depend on small continuous deformations of that area. This flux instead characterizes the gauge potential globally: it determines the first Chern characteristic class the gauge potential $W^{(0)}$ belongs to. An electron encircling the origin naturally acquires a topological phase associated with that nontrivial flux: the Aharonov-Bohm phase which distinguishes the gauge potential $W^{(0)}$ from a trivial one.

In the polar coordinates $(r, \varphi) \in R^{2}$ a plane can be regarded as an embedding

$$
(r, \varphi) \rightarrow(r \cos \varphi, r \sin \varphi, 0), \quad 0<r<\infty, 0 \leq \varphi<2 \pi .
$$

The gauge potential takes the form

$$
W_{r}^{(0)}=0, W_{\varphi}^{(0)}=-\nu
$$

The components of the induced metric ([6] can be easily read off

$$
g_{r r}=1, \quad g_{\varphi \varphi}=\alpha^{2} r^{2}, \quad g_{r \varphi}=g_{\varphi r}=0
$$

Evidently, this is a metric of a cone (cf. (11)) which at $\nu=0$ goes over to a flat one.

In general case the potential

$$
W_{a}^{(0) ;\left(\nu_{1}, \ldots, \nu_{N}\right)}(\vec{r})=-\sum_{i}^{N} \nu^{(i)} \frac{\epsilon_{a b}\left(\vec{r}-\vec{r}_{i}\right)^{b}}{\left|\vec{r}-\vec{r}_{i}\right|^{2}}
$$

where $a, b=x, y$, is supposed to describe $N$ disclination defects with the strengths $\nu^{(i)}$ located at the points with the coordinates $\vec{r}_{i}, i=1, . ., N$. To specify to an important in applications case of the disclination dipole located on the $x$-axis, we put $\vec{r}_{1}=(-L, 0), \vec{r}_{2}=(L, 0)$ and $\nu^{(1)}=-\nu^{(2)}=\nu$. The above equation then yields

$$
W_{x}^{(0)}=\nu \frac{4 x y L}{r_{+}^{2} r_{-}^{2}}, \quad W_{y}^{(0)}=\nu \frac{2 L\left(y^{2}-x^{2}+L^{2}\right)}{r_{+}^{2} r_{-}^{2}},
$$

where $r_{ \pm}^{2}=(x \pm L)^{2}+y^{2}$. Substituting this expression in Eq. (6) one can in principle compute the induced metric. To illustrate this, we work out an explicit representation of the metric induced by a disclination dipole in the asymptotic region $L / r \ll 1$. Far away from the dipole Eq.(12) reduces in the polar coordinates to

$$
W_{\varphi}^{(0)}=-2 \nu \varepsilon \cos \varphi+\mathcal{O}\left(\varepsilon^{2}\right), \quad W_{r}^{(0)}=\frac{2 \nu \varepsilon}{r} \sin \varphi+\mathcal{O}\left(\varepsilon^{2}\right) / r, \quad \varepsilon:=L / r \ll 1 .
$$

Our representation of the induced metric (6) then gives

$$
g_{r r}=1+\mathcal{O}\left(\varepsilon^{2}\right), \quad g_{\varphi \varphi}=r^{2}\left(1-4 \nu \varepsilon \cos \varphi+\mathcal{O}\left(\varepsilon^{2}\right)\right), \quad g_{r \varphi}=g_{\varphi r}=2 \nu \varepsilon r \sin \varphi+r \mathcal{O}\left(\varepsilon^{2}\right)
$$

This metric describes the asymptotic representation of the line element for a screw dislocation (see, e.g., 22]), with the Burgers vector $b^{y}=-4 \pi \nu L$ being perpendicular to the defect region. The gauge-theory approach is thus seen to recover a well-known result: at large distances from the defect a disclination dipole can be thought of as a screw dislocation.

\section{B. Incorporating fermions}

Let us now move on to a problem of coupling fermions to a given disclination. As is known, the topologically nontrivial gauge field reasserts itself in the Dirac equation as a topologically nontrivial piece of the spin connection [23]. That part of the connection carries a topologically nontrivial flux that does not depend on smooth continuous changes of the underlying metric due to small elastic deformations. To incorporate fermions on the $2 D$ background $\left(\Sigma_{0}=\right.$ $\left.R^{2}, \vec{r} \neq 0 ; g_{a b}^{(0)}\left(W^{(0)}\right)\right)$ we need a set of orthonormal frames $\left\{e_{\alpha}\left(W^{(0)}\right)\right\}$ which yield the same metric, $g_{a b}^{(0)}\left(W^{(0)}\right)$, related to each other by the local $S O(2)$ rotation,

$$
e_{\alpha} \rightarrow e_{\alpha}^{\prime}=\Lambda_{\alpha}^{\beta} e_{\beta}, \quad \Lambda_{\alpha}^{\beta} \in S O(2)
$$

It then follows that $g_{a b}=e_{a}^{\alpha} e_{b}^{\beta} \delta_{\alpha \beta}$ where $e_{\alpha}^{a}$ is the zweibein, with the orthonormal frame indices being $\alpha, \beta=\{1,2\}$, and coordinate indices $a, b=\{1,2\}$ (from now on we drop an explicit $W$-dependence of the metric). As usual, to 
ensure that physically observed values be independent of a particular choice of the zweinbein fields, a local so(2)valued gauge field $\omega_{\mu}$ is to be introduced. The gauge field of the local $S O(2)$ group is referred to as a spin connection. For the theory to be self-consistent, zweinbein fields must be chosen to be covariantly constant [24]:

$$
\partial_{a} e_{b}^{\alpha}-\Gamma_{a b}^{c} e_{c}^{\alpha}+\left(\omega_{a}\right)_{\beta}^{\alpha} e_{b}^{\beta}=0
$$

which determines the spin connection coefficients explicitly

$$
\left(\omega_{a}\right)^{\alpha \beta}=e_{b}^{\alpha} D_{a} e^{\beta b}, \quad D_{a}=\partial_{a}+\Gamma_{a},
$$

with $\Gamma_{a}$ being the Levi-Civita connection. The Dirac equation on a surface $\left(\Sigma_{0}, g_{a b}^{(0)}(W)\right)$ is written as

$$
i \gamma^{\alpha} e_{\alpha}^{a}\left(\partial_{a}+\Omega_{a}\right) \psi=E \psi
$$

with

$$
\Omega_{a}=\frac{1}{8} \omega_{a}^{\alpha \beta}\left[\gamma_{\alpha}, \gamma_{\beta}\right]
$$

being the spin connection in the spinor representation. In two space dimensions, the Dirac matrices can be chosen to be the Pauli matrices, $\gamma_{1}=-\sigma_{2}, \quad \gamma_{2}=-\sigma_{1}$. In the case under consideration Eq. (15) gives

$$
\omega_{r}^{12}=\omega_{r}^{21}=0, \quad \omega_{\varphi}^{12}=-\omega_{\varphi}^{21}=1-\alpha
$$

Hence, topologically nontrivial gauge field (9) results in a conical singularity of the spin connection. The flux

$$
\oint_{C} \omega_{\varphi}^{12} d \varphi=2 \pi \nu \neq 0
$$

represents a "net" effect produced by a disclination on the moving electrons. We thus show that the gauge-field approach in the inextensional limit exactly coincides with the standard "cut-and-glue" procedure.

However, a cone with a point-like apex is mathematical abstraction since in a real situation the media has a finite stiffness, which would inevitably result in a certain smearing of a conical singularity. Therefore, a proper description of the disclination implies a smooth deformation of the metric and at the same time one has to preserve a conical behavior far away from the origin. Although such a surface can effectively be approximated by a hyperboloid, we show now that one cannot incorporate finite elasticity into the theory by simply replacing a cone by a smooth surface that asymptotically approaches a cone far away from the origin. This would simply eliminate the defect.

To illustrate this, consider an upper half of a hyperboloid as an embedding

$$
(\xi, \varphi) \rightarrow(a \sinh \xi \cos \varphi, a \sinh \xi \sin \varphi, c \cosh \xi), \quad 0 \leq \xi<\infty, 0 \leq \varphi<2 \pi .
$$

The components of the induced metric can be written as

$$
g_{\xi \xi}=a^{2} \cosh ^{2} \xi+c^{2} \sinh ^{2} \xi, \quad g_{\varphi \varphi}=a^{2} \sinh ^{2} \xi, \quad g_{\varphi \xi}=g_{\xi \varphi}=0
$$

which in view of (15) gives for the spin connection coefficients

$$
\omega_{\xi}^{12}=\omega_{\xi}^{21}=0, \quad \omega_{\varphi}^{12}=-\omega_{\varphi}^{21}=\left[1-\frac{a \cosh \xi}{\sqrt{g_{\xi \xi}}}\right]=: \omega(\xi) .
$$

The spin connection in the spinor $S O(2)$ representation becomes

$$
\Omega_{\varphi}=i \omega \sigma_{3}
$$

Since $\omega(\xi)$ goes to zero as $\xi \rightarrow 0$ a circulation of that field over a loop encircling the origin gives a flux which tends to zero as the counter shrinks to zero,

$$
\lim _{\epsilon \rightarrow 0} \oint_{C_{\epsilon}} \omega_{\varphi}^{12} d \varphi=0
$$

where $C_{\epsilon}$ stands for a closed counter which encloses a small area $\sim \epsilon^{2}$ around the origin. This equation implies that there is no a topologically nontrivial part in the flux. It is therefore clear that one should work out some other way to explicitly accommodate elastic deformations in the "cut-and-glue" procedure that would preserve a conical singularity at the origin. 


\section{FINITE RIGIDITY}

In the present Section we formulate the linear elasticity theory in terms of the embeddings, which proves convenient to incorporate defects. We thus arrive at the von Karman equations to describe elastic disclinated media away from the limit of infinite rigidity. Our consideration allows then to introduce an important notion of the defect core radius, $r_{0}$. We assume that the linear theory works well in the region $r \geq r_{0}$ whereas it breaks down within the core, at $r<r_{0}$. We then briefly discuss the phenomenological approach discussed earlier to effectively account for the elastic properties of disclinated membranes [21]. Matching the linear von Karman theory at the boundary point $r=r_{0}$ with the phenomenological approach enables us to represent the phenomenological parameters in terms of the elasticity and bending constants.

\section{A. Elastic surface}

Let us start by discussing the elastic properties of a $2 D$ manifold in the absence of defects. Under elastic deformations a surface $\Sigma_{0}$ evolves into some other Riemannian surface $\Sigma$, which can be thought of as a diffeomorphic map, $\phi: \Sigma_{0} \rightarrow$ $\Sigma$. Again, we find it convenient to introduce the embedding $\Sigma \rightarrow R^{3}$ that can be realized in terms of a $R^{3}$-valued function $R^{i}\left(x^{1}, x^{2}\right)$, the point being that 25 ]

$$
\vec{R}(x):=\phi^{*} \vec{R}_{(0)}=\vec{R}_{(0)}[\phi(x)],
$$

where $\phi^{*}$ is a pullback of $\phi: \Sigma_{0} \rightarrow \Sigma$. The induced metrics becomes

$$
g_{a b} \equiv\left(\phi^{*} g_{\Sigma}\right)_{a b}=\left(g_{\Sigma}\right)_{c d} \frac{\partial \phi^{c}}{\partial x^{a}} \cdot \frac{\partial \phi^{d}}{\partial x^{b}}=\frac{\partial \vec{R}}{\partial \phi^{c}} \cdot \frac{\partial \vec{R}}{\partial \phi^{d}} \frac{\partial \phi^{c}}{\partial x^{a}} \cdot \frac{\partial \phi^{d}}{\partial x^{b}}=\partial_{a} \vec{R} \cdot \partial_{b} \vec{R}
$$

where the set $\left\{\phi^{a}\right\}$ stands for local coordinates on $\Sigma$. The strain tensor is then determined to be

$$
E_{a b}=g_{a b}-g_{a b}^{(0)} \text {. }
$$

The properties of a fluctuating elastic surface are encoded in the action

$$
F=F_{e l}+F_{f l},
$$

where $F_{e l}$ describes the elastic properties of the media, whereas $F_{f l}$ stands for the Helfrich-Canham action to describe the energy of a free fluctuating surface. Explicitly the stretching energy is taken to be quadratic in the strain,

$$
F_{e l}=-\frac{1}{8} \int_{\Sigma_{0}} d x^{1} d x^{2} \sqrt{g^{(0)}}\left\{\lambda(\operatorname{tr} E)^{2}+2 \mu \operatorname{tr} E^{2}\right\}
$$

where $\operatorname{tr} E=g_{(0)}^{a b} E_{a b}, g^{(0)}=\operatorname{det}\left\|g_{a b}^{(0)}\right\|$ and summation over repeated indices is assumed. Here $\lambda$ and $\mu$ are the $2 D$ Lame coefficients. The stress tensor is then introduced to be

$$
\sigma_{a b}=2 \mu E_{a b}+\lambda \operatorname{tr} E \delta_{a b} .
$$

The Helfrich-Canham bending energy of a membrane depends on its mean curvature $H$ and Gaussian curvature $K$ [26, 27],

$$
F_{f l}=\frac{\kappa}{2} \int_{\Sigma_{0}} \sqrt{g^{(0)}} d x^{1} d x^{2} H^{2}+\frac{\kappa_{G}}{2} \int_{\Sigma_{0}} \sqrt{g^{(0)}} d x^{1} d x^{2} K
$$

where $\kappa$ is a bare bending rigidity and $\kappa_{G}$ is a Gaussian rigidity. $H=g_{(0)}^{a b} K_{a b}$ is the mean (extrinsic) curvature, and $K=\operatorname{det}_{(0)}^{a b} K_{b c}$ is referred to as the Gaussian (intrinsic) curvature. Here

$$
K_{a b}=\vec{N} \cdot D_{a} D_{b} \vec{R}
$$

is the curvature tensor, and $\vec{N}$ is the unit normal to the surface

$$
\vec{N}=\frac{\left[\partial_{1} \vec{R}, \partial_{2} \vec{R}\right]}{\left|\left[\partial_{1} \vec{R}, \partial_{2} \vec{R}\right]\right|} .
$$

The covariant derivative

$$
D_{a}:=\partial_{a}+\Gamma_{a}
$$

includes the Levi-Civita connection $\Gamma_{a}$. 


\section{B. Disclinations in flexible membranes}

To incorporate disclinations originally distributed on $\Sigma_{0}$ one needs to make in Eq.(24) substitution

$$
\partial_{a} \vec{R}_{(0)} \rightarrow \nabla_{a} \vec{R}_{(0)}=\partial_{a} \vec{R}_{(0)}+\left[\vec{W}_{a}^{(0)}, \vec{R}_{(0)}\right]
$$

As a result, metric $g_{a b}^{(0)}$ goes over to $g_{a b}$ given by Eq.(6) . To derive equations of motion that follow from the Hamilton's principle of least action, $\delta F=0$, we first need to specify the embedding $R^{i}\left(x^{1}, x^{2}\right)$. In plane elasticity theory, an elastic deformation is represented by a displacement vector $u_{x}(x, y), u_{y}(x, y)$. If a membrane is allowed to buckle out of the plane, we must add an extra function $f(x, y)$ to describe the "deflection". We thus choose the embedding in the following way

$$
\vec{R}\left(x^{1}, x^{2}\right)=\vec{R}_{(0)}+\vec{U}
$$

where $\vec{R}_{(0)}=(x, y, 0)$ and $\vec{U}=\left(u_{x}, u_{y}, f(x, y)\right)$ is a displacement of the $(x, y, 0)$ point under deformation. In the linear approximation we may omit the terms quadratic in displacements $u_{a}$ as well as in the source strength $\nu$. The strain tensor then becomes

$$
E_{a b}=\partial_{a} u_{b}+\partial_{b} u_{a}+\partial_{a} f \partial_{b} f-\epsilon_{\alpha a} W_{b}^{(0)} R_{(0)}^{\alpha}-\epsilon_{\alpha b} W_{a}^{(0)} R_{(0)}^{\alpha}+\mathcal{O}\left(u^{2}, u \partial f, W^{2}\right) .
$$

Introducing the Airy stress function $\chi$,

$$
\sigma_{a b}=\epsilon_{a c} \epsilon_{b d} \partial_{c} \partial_{d} \chi
$$

one eventually gets the following equations of motion

$$
\begin{array}{r}
\kappa \Delta^{2} f=\left(\partial_{y}^{2} \chi\right)\left(\partial_{x}^{2} f\right)+\left(\partial_{x}^{2} \chi\right)\left(\partial_{y}^{2} f\right)-2\left(\partial_{x} \partial_{y} \chi\right)\left(\partial_{x} \partial_{y} f\right) \\
K_{0}^{-1} \Delta^{2} \chi=\left(\partial_{x} \partial_{y} f\right)^{2}-\left(\partial_{x}^{2} f\right)\left(\partial_{y}^{2} f\right)-\epsilon_{a b} \partial_{a} W_{b}^{(0)}
\end{array}
$$

where $K_{0}=4 \mu(\lambda+\mu) /(\lambda+2 \mu)$ is the $2 D$ Young's modulus.

A single disclination located at the origin of a plane is described by the potential (7). This results in

$$
-\epsilon_{a b} \partial_{a} W_{b}^{(0)}=\nu \Delta \log r=2 \pi \nu \delta(\vec{r})
$$

so that Eqs.(32) are exactly the von Karman equations for a defect in a flexible membrane [28]. Note, however, that the source term does not appear in (32) ad hoc but is rather generated by the gauge field due to a disclination. In case $N$ disclinations with strengthes $\nu_{i}, i=1,2, \ldots, N$ are located at points $\vec{r}_{i}$ one should use the gauge potential (11). The source term in Eq. (32) is then computed to be

$$
-\epsilon_{a b} \partial_{a} W_{b}^{\left(\nu_{1}, . . ., \nu_{N}\right)}=2 \pi \sum_{i}^{N} \nu_{i} \delta\left(\vec{r}-\vec{r}_{i}\right) .
$$

Dynamically induced metric on $\Sigma$ takes the form

$$
g_{a b}=\delta_{a b}+\partial_{a} u_{b}+\partial_{b} u_{a}+\partial_{a} f \partial_{b} f
$$

Both functions $\chi$ and $f$ or, equivalently, $u_{a}$ and $f$ contribute to it. Besides, function $f(x, y)$ determines a shape of the emergent surface. To see this, let us reexamine the case of a disclination on a plane that can be bent but cannot be stretched considered in Section II (see Eq.(10)). Within our approach it corresponds to the case of $K_{0} \rightarrow \infty$. Since there is no in-plane stretching one may put $u_{a}=0$ in Eqs.(32). The second von Karman equation takes the form

$$
\left(\partial_{x} \partial_{y} f\right)^{2}-\left(\partial_{x}^{2} f\right)\left(\partial_{y}^{2} f\right)=-2 \pi \nu \delta(\vec{r})
$$

whereas the induced metric becomes,

$$
g_{a b}=\delta_{a b}+\partial_{a} f \partial_{b} f
$$

Equation (34) possesses an obvious solution $f= \pm \sqrt{2 \nu} r$, which is a defining equation of a cone. The metric (35) coincides with that given by Eq. (10) up to $\mathcal{O}\left(\nu^{2}\right)$ order as it should in the linear approximation. 


\section{Solution to the von Karman equations}

It is instructive to reveal a geometrical structure of Eq.(34). The embedding (30) tells us that for an infinite stiffness the structure of the surface $\Sigma$ is entirely determined by function $z=f(x, y)$. In particular, the Gaussian curvature of the surface equals

$$
K=\frac{\left(\partial_{x}^{2} f\right)\left(\partial_{y}^{2} f\right)-\left(\partial_{x} \partial_{y} f\right)^{2}}{\left(1+\left(\partial_{x} f\right)^{2}+\left(\partial_{y} f\right)^{2}\right)^{2}}
$$

It is clear that a general solution to Eq.(34) must scale as $f \sim \sqrt{\nu}$. In the linear approximation one can therefore drop the $f$-dependent terms in the denominator in (36), whereupon that equation takes the form

$$
K=2 \pi \nu \delta(\vec{r})
$$

This means that all the curvature is located in this case at the apex. Thus, to get the curvature spread over some finite area one needs to take into consideration elastic properties of a media. In other words, one needs to consider the whole set of the von Karman equations (32) at finite elasticity and bending constants.

To this end, let us first rewrite the von Karman equations in the dimensionless form. Under the substitutions $\chi \rightarrow \chi \kappa, \vec{r} \rightarrow \vec{r} r_{0}$ and $f \rightarrow f r_{0}$ where $r_{0}$ is yet unspecified parameter with the dimension of length, those equations become

$$
\begin{aligned}
\Delta^{2} f & =\left(\partial_{y}^{2} \chi\right)\left(\partial_{x}^{2} f\right)+\left(\partial_{x}^{2} \chi\right)\left(\partial_{y}^{2} f\right)-2\left(\partial_{x} \partial_{y} \chi\right)\left(\partial_{x} \partial_{y} f\right) \\
\epsilon \Delta^{2} \chi & =2 \pi \nu \delta(\vec{r})-K
\end{aligned}
$$

where the parameter $\epsilon=\kappa /\left(K_{0} r_{0}^{2}\right)$ and the Gaussian curvature $K=\operatorname{det}\left(\partial_{a} \partial_{b} f\right)$. All the functions as well as the coordinates entering these equations are now dimensionless. Since in $2 D$ one gets $\left[K_{0}^{1 / 3}\right]=[\kappa]=E$, the parameter $\epsilon$ is dimensionless as well. The inextensional limit amounts to that of $\epsilon \rightarrow 0$. Let us denote the solutions to the von Karman equations in this limit by $f_{0}$ and $\chi_{0}$. The second line in Eqs.(38) becomes

$$
\left(\partial_{x}^{2} f_{0}\right)\left(\partial_{y}^{2} f_{0}\right)-\left(\partial_{x} \partial_{y} f_{0}\right)^{2}=2 \pi \nu \delta(\vec{r})
$$

which has an obvious solution $f_{0}= \pm \sqrt{2 \nu} r$ that describes as already mentioned a true cone. Inserting $f_{0}$ into the first equation of (38) gives

$$
1=\left(x^{2} \partial_{x x}^{2}+y^{2} \partial_{y y}^{2}+2 x y \partial_{x y}^{2}\right) \chi_{0}
$$

This equation possesses an obvious inhomogeneous solution $\chi_{0}=-\log r$ discussed at length in [28]. However, there is also a nontrivial solution of the corresponding homogeneous equation missed in [28]. A general solution to Eq. (39]) must include a homogeneous term and reads

$$
\chi_{0}=-\log r+q r
$$

Here $q$ is an arbitrary constant. This becomes evident upon rewriting Eq. (39) in the polar coordinates, $1=r^{2} \partial_{r r}^{2} \chi_{0}$. The homogeneous part of the solution (40) turns out to be of the utmost importance in stabilizing the theory and bringing out the physical meaning of the parameter $r_{0}$.

To see this, let us for a moment restore an explicit $r_{0}$ dependence in Eq. (40):

$$
\chi_{0}=-\log \frac{r}{r_{0}}+q \frac{r}{r_{0}} .
$$

Consider an elastic thin disk of the radius $R$ with a disclination sitting at the origin. Because of rotational symmetry,

$$
\sigma_{r \varphi}=-\partial_{r}\left(\frac{1}{r} \partial_{\varphi} \chi\right)
$$

vanishes identically for (41). The radial component of the strain tensor

$$
\sigma_{r r}=\frac{1}{r} \partial_{r} \chi+\frac{1}{r^{2}} \partial_{\varphi \varphi}^{2} \chi
$$


yields, however

$$
\sigma_{r r}=-\frac{1}{r^{2}}+\frac{q}{r r_{0}}
$$

It is clear that $\sigma_{r r}(r=R)$ vanishes as $R \rightarrow \infty$. It is singular, however, at the origin. Moreover, if we ignored the homogeneous solution in (42) we would run into a serious problem. Namely, a typical way to remove the singularity is to delete a small disk of material around the origin. It is easy to see that for $q=0$ the strain tensor $\sigma_{r r}$ behaves like $-1 / r^{2}$ at the boundary of the excised disk. This is clearly physically unacceptable, which signals the instability of the theory (see discussion in [28]). It should be stressed that the general solution (41) allows us to avoid this difficulty. To show this, let us delete a small disk of the radius $r_{0}$ around the origin and require that $\sigma_{r r}\left(r=r_{0}\right)=\sigma_{0}$. This yields $q=1+\sigma_{0} r_{0}^{2}$. If one requires vanishing stresses at the inner boundary, one should put $\sigma_{0}=0$. Therefore, the parameter $r_{0}$ characterizes the core region of the disclination.

It is also important to note that the homogeneous term in the solution (42) significantly affects the stretching energy of the membrane,

$$
E_{s}=\frac{1}{2 K_{0}} \int_{r>r_{0}} d^{2} \vec{r}\left(\nabla^{2} \chi\right)^{2}
$$

Since $\nabla^{2} \log r=2 \pi \delta(\vec{r})$ the stretching energy (43) for the stress function (42) with $q=0$ becomes an identical zero. This is exactly the conclusion reached in [28]. However, this is not physically appropriate, since this result should follow only in the limit $K_{0} \rightarrow \infty$. If we instead compute (43) at $q \neq 0$, we will get

$$
E_{s} \propto \frac{\kappa^{2} q^{2}}{K_{0} r_{0}^{2}} \log \frac{R}{r_{0}}=\epsilon \kappa q^{2} \log \frac{R}{r_{0}},
$$

which indeed vanishes as $K_{0} \rightarrow \infty$. It should be stressed that the same logarithmic behavior has the bending energy, which is written as 28]

$$
E_{b}=2 \pi \nu \kappa \log \frac{R}{r_{0}}
$$

At $q \sim 1$ one has $E_{s} \sim \epsilon E_{b}$ as it should be at small $\epsilon$. Because of the fact that the entropy in the Kosterlitz-Thouless argument also increases logarithmically, this result provides an interesting possibility of disclination-mediated phase transitions that might be realized in $2 D$ elastic membranes (see, e.g., [29]).

\section{Phenomenological approach vs von Karman equations}

Let us now turn back to the dimensionless set of the von Karman equations (38). We seek a general solution in the form

$$
f=\sum_{n=0}^{\infty} \epsilon^{n} f_{n}, \quad \chi=\sum_{n=0}^{\infty} \epsilon^{n} \chi_{n},
$$

where we have already found $f_{0}$ and $\chi_{0}$. These series are supposed to converge, provided the linear approximation is valid. Inserting (46) back into (38) results in a set of the self-consistent coupled equations to determine step by step the functions $f_{n}$ and $\chi_{n}$. Technically, those equations for $n \geq 1$ turn out to be quite complicated. Their analysis will be given elsewhere. To get some insight, we invoke instead a sort of phenomenological approach to effectively incorporate elasticity in the Dirac equation discussed earlier in [21]. We match the information that follows from our approach with that provided by the the von Karman equations. This enables us to explicitly determine the dependence of the phenomenological parameters on the elasticity and bending constants to analyze the electronic properties of elastic graphene.

To begin with, let us briefly recall the phenomenological theory [21]. As was already mentioned, a rigid plane pierced by a vortex results in a conical singularity. Let us now assume that the membrane possesses a small finite elasticity. In that case the vortex will produce the singularity at the origin as before and, additionally, it causes the medium to respond by smoothing the conical shape due to elasticity. We suggest that both effects can be taken into account by placing the vortex on the tip of a hyperboloid of a near-zero curvature. That sort of hyperboloid is supposed to effectively emerge as a response of the elastic plane to a disturbance caused by the defect at large distances, $r>r_{0}$. The parameters of the hyperboloid must fulfil some natural requirements to be formulated shortly. 
In this way we arrive at the effective metric that takes into account a response of the elastic media to the disturbance caused by the defect.

Explicitly, we employ the embedding (19), with dimensionless (scaled by $r_{0}$ ) parameters $a$ and $c$ that appear as the phenomenological parameters of the theory. The gauge field (9) represents the vortex at the origin. It induces the following metric:

$$
g_{\xi \xi}=a^{2} \cosh ^{2} \xi+c^{2} \sinh ^{2} \xi, \quad g_{\varphi \varphi}=a^{2} \alpha^{2} \sinh ^{2} \xi, \quad g_{\varphi \xi}=g_{\xi \varphi}=0
$$

where $\alpha=1-\nu$ and $\nu$ is the declination charge. At $\nu=0$ this metric reduces to that of a true hyperboloid given by (20). Comparing the embedding (19) with Eq.(30) tells us that $c$ must scale as $\sqrt{\nu}$, which seems natural: buckling is induced by the source. Let us further suggest that the elasticity coefficients are accumulated in parameter $a$, the inextensional limit corresponding to $a \rightarrow \infty$. In other words, we should get that $a \rightarrow \infty$ as $K_{0} \rightarrow \infty$. Let us check these two assumptions against the metric (47). We see that at $\nu=0$ it becomes

$$
g_{\xi \xi}=a^{2} \cosh ^{2} \xi, \quad g_{\varphi \varphi}=a^{2} \sinh ^{2} \xi, \quad g_{\varphi \xi}=g_{\xi \varphi}=0 .
$$

Upon introducing a new variable $r=a \sinh \xi$ one gets

$$
g_{r r}=g_{\xi \xi}(r)\left(\frac{\partial \xi}{\partial r}\right)^{2}=1, \quad g_{\varphi \varphi}=r^{2}, \quad g_{\varphi r}=g_{r \varphi}=0,
$$

which is nothing but the metric of a plane. This result is quite reasonable, since putting $\nu=0$ should result in the trivial solution to the von Karman equations, $f=\chi=0$. In the second case of interest, we have $\nu \neq 0$ and $a \gg 1$. With the help of the above mentioned change of variables, we get

$$
g_{r r}=1+\left(\frac{c}{a}\right)^{2} \frac{r^{2}}{a^{2}+r^{2}}, \quad g_{\varphi \varphi}=\alpha^{2} r^{2}, \quad g_{\varphi r}=g_{r \varphi}=0 .
$$

We see that for large enough $a$, which in view of our assumption implies large $K_{0}$, the elasticity properties come into play through the single dimensionless parameter $\eta=c^{2} / a^{2} \ll 1$. In particular, in the inextensional limit $a \rightarrow \infty$, Eq.(50) reduces to the metric of a cone.

We now argue that the desired form of $\eta$ follows directly from the von Karman equations. To this end we suppose that there exist two dimensionless functions $\tilde{\chi}$ and $\tilde{f}=f_{0}+\tilde{f}$ that fulfil Eqs. (38) and at the same time account for the embedding (19) as well as metric (47). We have explicitly singled out the $f_{0}$ dependence to eliminate the $\delta$-function source. We are interested in the second "dynamical" equation in Eq. (38). It takes the form

$$
\epsilon \Delta^{2} \tilde{\chi}=-K\left(f_{0}, \tilde{f}\right), \quad r \geq 1 .
$$

To proceed we simply replace the Gaussian curvature in this equation with that of the hyperboloid

$$
K=\frac{c^{2}}{\left(a^{2}+r^{2}(1+\eta)\right)^{2}} .
$$

We recall that coordinate $r$ as well as parameters $a$ and $b$ are now dimensionless. Equating then the both sides of (51) at the core boundary $r=1$ yields

$$
\eta^{2}=k c^{2} \epsilon+\mathcal{O}\left(\epsilon^{2}\right) .
$$

In getting this we have assumed that $a \gg 1$. Here $k=\left.\Delta^{2} \tilde{\chi}\right|_{r=1}$. To restore an explicit $r_{0}$-dependence one needs to make the substitution $a \rightarrow a / r_{0}, c \rightarrow c / r_{0}$. This yields

$$
\eta^{2}=k\left(c / r_{0}\right)^{2} \epsilon .
$$

It seems reasonable to assume that $c \sim \sqrt{\nu} r_{0}$, which finally gives

$$
\eta \sim \sqrt{\nu \epsilon}
$$

Eq. (55) qualitatively relates the characteristics of elastic media to the parameters of the embedding. It must be stressed that the smooth hyperboloid parametrized by Eq.(19) at $\nu=0$ is not a surface that replaces a rigid cone for finite elasticity. Only when that hyperboloid of the near-zero curvature is pierced by the gauge flux, the smoothed replacement of the cone emerges. By construction $a \gg r_{0}$ and $c \sim r_{0}$, which is consistent with $\eta \ll 1$. This takes care of a small stretching in the system. 
Turning back to fermions, we see that the metric (47) generates the spin connection term

$$
\omega_{\varphi}^{12}=-\omega_{\varphi}^{21}=\left[1-\frac{a \alpha \cosh \xi}{\sqrt{g_{\xi \xi}}}\right]=\omega_{\alpha}(\xi)
$$

Since $\omega_{\alpha}(\xi) \rightarrow 1-\alpha$ as $\xi \rightarrow 0$, it in contrast with (21) contains a topologically nontrivial part that gives rise to a fixed flux,

$$
\lim _{\epsilon \rightarrow 0} \oint_{C_{\epsilon}} \omega_{\varphi}^{12} d \varphi=2 \pi \nu
$$

We thus get the smoothed apex, the cone-like asymptotic at large distances and the unremovable conical singularity at the disclination line. It is known that in case a spin connection contains an $S O(2)$ piece with nontrivial flux, that field cannot be eliminated under any smooth deformation of the underlying metric (see, e.g., [24]). Within our approach this simply means that a nontrivial contribution to the spin connection which comes from the topological gauge field survives any smooth elastic deformations of the media.

\section{FLEXIBLE GRAPHENE}

In this section, we apply the developed approach to describe the electronic properties of graphene with pentagonal defects. Indeed, elastic characteristics of graphene are well-fitted to our theory. The estimated bending rigidity of graphene lies in the range of $1-2 \mathrm{eV}$ and anyway does not exceed the value of $2.5 \mathrm{eV}$ (see, e.g., 30 32]). At the same time, the lower range value of $K_{0} r_{0}^{2}$ is approximately given by $20 \mathrm{eV}$ at $r_{0}=a_{0}$ with $a_{0}$ being the interatomic spacing in graphene lattice [30]. Therefore, the parameter $\epsilon$ is estimated as $\epsilon \leq 0.1$ thus justifying an applicability of the elasticity-induced perturbation scheme to graphene.

\section{A. Uniform magnetic field: Landau states}

The Dirac equation on a surface $\Sigma$ in the presence of the gauge field $a_{b}$ and the external magnetic field with the vector potential $A_{b}$ is written as

$$
i \gamma^{\alpha} e_{\alpha}^{b}\left[\nabla_{b}-i a_{b}-i A_{b}\right] \psi=E \psi
$$

where $\nabla_{b}=\partial_{b}+\Omega_{b}$. The effective abelian gauge field $a_{b}$ is responsible for valley mixing since $K$ and $K^{\prime}$ points become inequivalent in the presence of the pentagonal defect (see, e.g., [1] for detail). The energy in (57) is measured from the Fermi level.

On a surface of the hyperboloid the Dirac operator reads

$$
\hat{D}=\left(\begin{array}{cc}
0 & e^{-i \varphi}\left(-\frac{\partial_{\xi}}{\sqrt{g_{\xi \xi}}}+\frac{1}{a \alpha \sinh \xi}\left(i \partial_{\varphi}+\frac{1}{2} \omega_{\alpha}(\xi)+\Omega_{\varphi}\right)\right) \\
e^{i \varphi}\left(\frac{\partial_{\xi}}{\sqrt{g_{\xi \xi}}}+\frac{1}{a \alpha \sinh \xi}\left(i \partial_{\varphi}-\frac{1}{2} \omega_{\alpha}(\xi)+\Omega_{\varphi}\right)\right) & 0
\end{array}\right)
$$

where

$$
\Omega_{\varphi}=a_{\varphi}+A_{\varphi}
$$

It can be verified that $\hat{D}=\hat{D}^{\dagger}$. The substitutions

$$
\left(\begin{array}{c}
\psi_{A} \\
\psi_{B}
\end{array}\right)=\frac{1}{\sqrt{2 \pi}}\left(\begin{array}{c}
u(\xi) e^{i j \varphi} \\
v(\xi) e^{i(j+1) \varphi}
\end{array}\right), \quad j=0, \pm 1, \ldots
$$

and

$$
\tilde{\psi}=\psi \sqrt{\sinh \xi},
$$

reduce the Dirac equation $\hat{D} \psi=E \psi$ to the form

$$
\partial_{\xi} \tilde{u}-\frac{\left(j+1 / 2-a_{\varphi}+A_{\varphi}\right)}{\alpha} \sqrt{\operatorname{coth}^{2} \xi+\eta \tilde{u}}=\tilde{E} \tilde{v},
$$




$$
-\partial_{\xi} \tilde{v}-\frac{\left(j+1 / 2-a_{\varphi}+A_{\varphi}\right)}{\alpha} \sqrt{\operatorname{coth}^{2} \xi+\eta} \tilde{v}=\tilde{E} \tilde{u}
$$

where $\tilde{E}=\sqrt{g_{\xi \xi}} E$. Explicitly, $a_{\varphi}= \pm(N / 4+M / 3)$ for even number of pentagons and $a_{\varphi}= \pm N / 4$ for odd number of pentagons. Here $N$ is the number of pentagonal defects and $M$ takes the values $M=-1,0,1$ depending upon the arrangement of pentagons (see [33] for detail). The uniform external magnetic field $B$ is chosen to be pointed in the $z$-direction so that $\vec{A}=B(y,-x, 0) / 2$. In $(\xi, \varphi)$ coordinates the components of $A_{b}$ are written as

$$
A_{\varphi}=-\Phi \sinh ^{2} \xi ; \quad A_{\xi}=0
$$

where $\Phi=b a^{2} / 2$ and $b=e B / \hbar c$. Let us start with the analysis of the electron state at the Fermi level (so-called zero-energy mode). In this case, we put $\mathrm{E}=0$ in (62) and (63). The exact solution is found to be

$$
\begin{aligned}
& \tilde{u_{0}}(\xi)=C(\Delta+k \cosh \xi)^{k \tilde{j}+\frac{\eta \tilde{\Phi}}{2 k}}\left(\frac{\Delta+\cosh \xi}{\sinh \xi}\right)^{-\tilde{j}} \exp \left(-\frac{\tilde{\Phi} \Delta \cosh \xi}{2}\right), \\
& \tilde{v_{0}}(\xi)=C^{\prime}(\Delta+k \cosh \xi)^{-k \tilde{j}-\frac{\eta \Phi}{2 k}}\left(\frac{\Delta+\cosh \xi}{\sinh \xi}\right)^{\tilde{j}} \exp \left(\frac{\tilde{\Phi} \Delta \cosh \xi}{2}\right),
\end{aligned}
$$

where

$$
k=\sqrt{1+\eta} ; \quad \Delta=\Delta(\xi)=\sqrt{1+k^{2} \sinh ^{2} \xi}, \quad \tilde{j}=\left(j+1 / 2-a_{\varphi}\right) / \alpha, \quad \tilde{\Phi}=\Phi / \alpha
$$

and $C$ and $C^{\prime}$ are the normalization factors. Evidently, the only component $u_{0}$ becomes normalizable. In the inextentional limit $(\eta \rightarrow 0)$ one obtains

$$
u_{0}(r) \propto r^{\tilde{j}-1 / 2} \exp \left(-\frac{b r^{2}}{4 \alpha}\right)
$$

where $r=a \sinh \xi$. This result agrees with that of [33].

Let us consider the Landau states. To this end, we develop the perturbation scheme using $\eta \sim \nu \epsilon$ as the perturbation parameter. Indeed, according to (50) the hyperboloid can at small $\eta$ be considered as a local perturbation of the cone metric. Notice that a similar procedure was used in the description of electronic states in spheroidal fullerenes [34, 35] where the spheroid was considered as a slightly elliptically deformed sphere. At the same time, the Landau states on the graphene cone were already studied in detail in [33]. Therefore, one can use the unperturbed solutions found there.

The Dirac operator is written as

$$
\hat{\mathcal{D}}=\hat{\mathcal{D}}_{0}+\eta \hat{\mathcal{D}}_{1}
$$

where

$$
\hat{\mathcal{D}}_{0}=i \gamma_{2} \frac{1}{a \cosh \xi}\left(\partial_{\xi}+\frac{\cosh \xi}{2 \sinh \xi}\right)-\frac{\gamma_{1}}{a \sinh \xi}\left(\tilde{j}+\tilde{\Phi} \sinh ^{2} \xi\right)
$$

is the Dirac operator on the true cone. It is important to note here that generally the operator $\hat{\mathcal{D}}_{1}$ is not Hermitian on a cone and must be extended to a Hermitian one (see, e.g., the discussion in [34, 35]). The result is

$$
\hat{\mathcal{D}}_{1}=-\frac{\gamma_{1} \sinh \xi}{2 a \cosh ^{2} \xi}\left(\tilde{j}+\tilde{\Phi} \sinh ^{2} \xi\right) .
$$

It is convenient to square the Dirac operator,

$$
\hat{\mathcal{D}}^{2}=\hat{\mathcal{D}}_{0}^{2}+\eta \hat{\Gamma}
$$

where $\hat{\Gamma}=\hat{\mathcal{D}}_{0} \hat{\mathcal{D}}_{1}+\hat{\mathcal{D}}_{1} \hat{\mathcal{D}}_{0}$ and the quadratic in $\eta$ term is omitted. Explicitly,

$$
\hat{\Gamma}=\frac{1}{a^{2} \cosh ^{2} \xi}\left[\left(\tilde{j}+\tilde{\Phi} \sinh ^{2} \xi\right)^{2}+\frac{\sigma_{3}}{2}\left(\tilde{j}\left(\frac{2}{\cosh ^{2} \xi}-1\right)+\tilde{\Phi} \sinh ^{2} \xi\left(\frac{2}{\cosh ^{2} \xi}+1\right)\right)\right] .
$$


For $\eta=0$ both the unperturbed wave functions $\psi_{j n}$ and the Landau energy levels were obtained in [33]. Two families of solutions were found. We restrict our consideration here to the first family where $\tilde{j} \geq 0$. In this case, the energy of the so-called bulk levels reads [33] $E_{n}^{0}= \pm \sqrt{2 n}, n=0,1,2 \ldots$, where the energy is measured in units of $\hbar v_{F} / l_{B}$ with the magnetic length $l_{B}=\sqrt{\hbar c / e B}$.

Let us calculate the matrix element of the perturbation $\Gamma=\left\langle\psi_{j n}|\hat{\Gamma}| \psi_{j n}\right\rangle$. Our analysis shows that the perturbation does not influence the zero energy level in the first order in $\eta$. Since the resulting expressions are rather involved, we calculate the first energy level numerically. The first Landau level is found to be slightly shifted due to the elastic contribution,

$$
E_{n=1}^{\eta} \simeq \pm \sqrt{2} \pm \eta \frac{\Gamma}{2 \sqrt{2}} \simeq \pm \sqrt{2} \pm 0.3 \eta, \quad \eta>0,
$$

where the \pm sign corresponds to the conduction and valence band, respectively. Note that a similar shift of the first Landau level follows for the second $(\tilde{j} \leq 0)$ family of the solutions referred to as the apical states 33 .

In graphene there are many allowed transitions due to the presence of two electron bands, the conduction and the valence band, and the transitions have the energies

$$
\Delta_{n+1, n}^{\xi}=\frac{\hbar v_{F}}{l_{B}}[\sqrt{2(n+1)}-\xi \sqrt{2 n}]
$$

where $\xi= \pm$ denote the intraband and interband transitions, respectively [36]. As is seen from Eq.(71) the transition between the ground energy level and the first one in the presence of elasticity is modified to become

$$
\Delta_{1,0}=\frac{\hbar v_{F}}{l_{B}}[\sqrt{2}+0.3 \eta]
$$

The experimentally observed cyclotron resonancelike and electron-positron-like transitions are in a good agreement with the theoretical expectations of a single-particle model of Dirac fermions in graphene [37]. They produce a very accurate value for $v_{F}$, the velocity of electrons in graphene. In our case, the Fermi velocity becomes slightly renormalized due to the elasticity effects induced by the phenomenological parameter $\eta: \Delta v_{F} / v_{F}=0.3 \eta / \sqrt{2}$. Therefore, it would be interesting to perform analogous experiments with graphite cones instead of graphene to determine the modification of $v_{F}$.

\section{B. Zero magnetic field: Electronic density of states}

Let us consider now the case of zero magnetic field, $B=0$. This markedly changes the situation with the zero-mode states. Indeed, in the absence of the cut-off exponent in (65) and (66) the normalization conditions are found to be $-1 / 2<\tilde{j}<-1 / 2 k$ for $u_{0}(\xi)$ and $1 / 2 k<\tilde{j}<1 / 2$ for $v_{0}(\xi)$. As a result, at small $\eta$ which is of interest here, there are no normalized solutions. This means that smoothing has no marked effect on the existence of zero modes. As stated above, one of the modes (either $\tilde{u}(\xi)$ or $\tilde{v}(\xi)$ ) becomes in the presence of the uniform magnetic field directed along the $z$-axis nonmailable and there exists a true zero mode. Therefore, one can expect a "switching-like" effect driven by the magnetic field.

An interesting question is how a hyperboloid geometry influences the density of states near the Fermi energy in the vicinity of the pentagonal defects. We will be interested in the DOS in a small ring $0<r \leq \delta$ around the defects. First, we need to find corrections to the wave functions for the hyperboloid geometry. Following the perturbation scheme with $\eta$ being a small parameter, one can write $\psi_{n}=\psi_{n}^{0}+\psi_{n}^{\eta}+\ldots$ with $\psi_{n}^{0}$ being the solutions for $\eta=0$ which are the ordinary Bessel functions (see [8]). Here $\psi_{n}^{\eta} \propto \sum_{m} \Gamma_{m n} \psi_{m}^{0} / \Delta k$ is a perturbative part with $\Delta k=\pi / R$. The matrix element of the perturbation reads $\Gamma_{m n}=\left\langle\psi_{j m}^{0}|\hat{\Gamma}| \psi_{j n}^{0}\right\rangle$, where the perturbation term takes the form

$$
\hat{\Gamma}=\frac{\tilde{j}}{a^{2} \cosh ^{2} \xi}\left[\tilde{j}+\frac{\sigma_{3}}{2}\left(\frac{2}{\cosh ^{2} \xi}-1\right)\right] .
$$

Finally, the total DOS in the $\delta$ disk is found to be

$$
D(E, \delta, n) \propto D O S^{0}+\eta D O S^{\eta} \propto D O S^{0}\left(1+\eta\left|\frac{E}{a}\right| \delta^{2}\right), \quad \eta>0,
$$

where

$$
D O S^{0} \propto|E|^{2 n+1} \delta^{2(n+1)},
$$


coincides with the total DOS near the defects for graphitic cones found in [8], and $n=\tilde{j} \pm 1 / 2$ is the index of the Bessel function. The term

$$
D O S^{\eta} \propto|E|^{2(n+1)} \delta^{2(n+2)},
$$

comes from the elastic perturbation. Thus, we obtain an increase of DOS in the vicinity of the defect due to the finite elasticity. This behavior can be explained by the elasticity-induced contribution to the underlying metric which markedly deviates from that of a true cone near the apex. The modification of the DOS is more pronounced for more flexible membranes. Notice that the bending rigidity of square graphene is found to grow with a size [31] while the Young's modulus depends on the temperature [32]. These findings give a possibility of experimental studies of the influence of elasticity on the electronic characteristics of disclinated graphene. As a promising material, one can consider the graphene monolayers obtained via chemical reduction of graphene oxide which have excellent mechanical properties including high bending flexibility and tensile strength [38].

\section{CONCLUSION}

In conclusion, we have presented an analytical approach to describe Dirac fermions on a flexible disclinated surface beyond the inextensional limit. The elastic membrane is considered as an embedding of a $2 D$ surface into $R^{3}$. The disclination is incorporated through a topologically nontrivial $S O(2)$ gauge field that generates a metric with a conical singularity. A smoothing of the conical singularity is accounted for by regarding the upper half of a disclinated two-sheet hyperboloid as an elasticity-induced embedding. Parameters of that embedding are chosen to match the solution to the von Karman equations. Away from but close to the inextensional limit, the Young's modulus $K_{0}$ and bending rigidity $\kappa$ enter the theory through a dimensionless parameter, $\epsilon=\kappa /\left(K_{0} r_{0}^{2}\right) \ll 1$, where $r_{0}$ sets the relevant short-range scale: a radius of the defect core.

We argue that the homogeneous part of the solution to the von Karman equations for disclinated membrane is of the utmost importance in stabilizing the theory. This finding allows us to avoid the evident problem with the core radius mentioned in [28]. Surprisingly, the discovered homogeneous part of the solution has been missed so far. We calculate the stretching energy of the membrane to observe the logarithmic behavior with the membrane radius $R$ similar to that of the bending energy. For rigid enough membranes we work out the self-consisting perturbation scheme with $\epsilon$ being the small parameter.

We apply a new approach to study the structure of the low energy electronic states of flexible graphene with a topological defect. We find a true zero-mode state in the presence of an external uniform magnetic field. The finite elasticity results in a smearing of the cone apex thus modifying the cone metric. The elasticity affects enter the observable quantities through the elasticity-induced phenomenological parameter $\eta \sim \sqrt{\nu \epsilon}$. Qualitatively, the first Landau level in the conduction band is found to be shifted upwards, whereas the corresponding one in the valence band is shifted downwards. The total DOS near the tip is shown to increase due to elasticity.

From the experimental point of view, it would be interesting to carry out experiments with elastic graphite cones to determine the modification of $v_{F}$ due to elastic deformations caused by a disclination defect. The elasticity effects are more significant for more flexible materials. Therefore graphene-based materials with excellent mechanical properties like those recently obtained via chemical reduction of graphene oxide in [38] would be of interest in these studies.

ACKNOWLEDGEMENTS -E.A.K. and V.A.O. acknowledge financial support by the Russian Foundation for Basic Research under grant No. 08-02-01027. R.P. acknowledges financial support by the Slovak Academy of Sciences in the framework of CEX NANOFLUID, and by the Science and Technology Assistance Agency under Contract No. APVV 0509-07 and by VEGA Grant No. 2/0069/10.

[1] A.H. Castro Neto, F. Guinea, N.M.R. Peres, K.S. Novoselov, and A.K. Geim, Rev. Mod. Phys. 81 (2009) 109.

[2] A. Cortijo and M.A.H. Vozmediano, Europhys. Lett. 77 (2007) 47002.

[3] J.K. Pachos, Cont. Phys. 50 (2009) 375.

[4] B. An, S. Fukuyama, K. Yokogawa, M. Yoshimura, M. Egashira, Y. Korai, and I. Mochida, Appl. Phys. Lett. 78 (2001) 3696.

[5] J. -C. Charlier and G. -M. Rignanese, Phys.Rev.Lett. 86 (2001) 5970.

[6] J. González, F. Guinea and M.A.H. Vozmediano, Phys. Rev. Lett. 69 (1992) 172.

[7] J. González, F. Guinea and M.A.H. Vozmediano, Nucl.Phys. B 406 (1993) 771.

[8] P.E. Lammert and V.H. Crespi, Phys. Rev. Lett. 85 (2000) 5190.

[9] V.A. Osipov and E.A. Kochetov, JETP Lett. 72 (2000) 199. 
[10] V.A. Osipov and E.A. Kochetov, JETP Lett. 73 (2001) 631.

[11] J.K. Pachos, A. Hatzinikitas, and M. Stone, Eur.Phys. J. Special Topics 148 (2007) 127.

[12] T. Ando, Prog. Theor. Phys., Suppl. 176 (2008) 203.

[13] C. Chamon, C.Y. Hou, R. Jackiw, C. Mudry, S.Y. Pi, and G. Semenoff, Phys. Rev. B 77 (2008) 235431.

[14] A. Mesaros, D. Sadri, and J. Zaanen, Phys. Rev. B 79 (2009) 155111.

[15] Yu.A. Sitenko and N.D. Vlasii, Nucl. Phys. B 787 (2007) 241.

[16] V.M. Pereira and A.H. Castro Neto, Phys. Rev. Lett. 103 (2009) 046801.

[17] F. Guinea, M.I. Katsnelson, and A.K. Geim, Nature Physics 6 (2010) 30.

[18] B. Allen and A.C. Ottewill, Phys. Rev. D 42 (1990) 2669.

[19] B. Allen, B.S. Kay, and A.C. Ottewill, Phys. Rev. D 53 (1996) 6829.

[20] C.A. de Lima Ribeiro, C. Furtado, and F. Moraes, Phys. Lett. A 288 (2001) 329.

[21] E.A. Kochetov and V.A. Osipov, JETP Lett. 91 (2010) 110.

[22] R.A. Putingam and H.H. Soleng, Class. Quantum Grav. 14 (1997) 1129.

[23] V.A. Osipov, E.A. Kochetov, and M. Pudlak, JETP 96 (2003) 140.

[24] M.B. Green, J.H. Schwartz, and E. Witten, Superstring theory, (Cambridge 1988), v. 2.

[25] E.A. Kochetov and V.A. Osipov, J.Phys. A: Math.Gen. 32 (1999) 1961.

[26] W. Helfrich Z. Naturforsch. C 28 (1973) 693.

[27] P. Canham J. Teo. Bio. 26 (1970) 61.

[28] H.S. Seung and D.R. Nelson, Phys.Rev. A 38 (1988) 1005.

[29] D.R. Nelson, in Phase Transitions and Critical Phenomena, eds. Domb and J. Lebowitz, Vol. 7 (Academic Press, London) 1983, pp. 1-99.

[30] K. N. Kudin, G. E. Scuseria, and B. I. Yakobson, Phys. Rev. B 64 (2001) 235406.

[31] Q. Wang, Phys. Lett. A 3741180 (2010).

[32] K. V. Zakharchenko, M. I. Katsnelson and A. Fasolino, Phys. Rev. Lett. 102 (2009) 046808.

[33] P. E. Lammert, V. H. Crespi, Phys. Rev. B 69 (2004) 035406.

[34] M. Pudlak, R. Pincak and V.A. Osipov, Phys. Rev. B 74 (2006) 235435.

[35] M. Pudlak, R. Pincak and V.A. Osipov, Phys. Rev. A 75 (2007) 025201.

[36] Mark O. Goerbig, "Quantum Hall Effects", Lecture notes for the Singapore session "Ultracold Gases and Quantum Information" of Les Houches Summer School, (2009).

[37] M.L. Sadowski, G. Martinez, M. Potemski, C. Berger, and W.A. de Heer, Phys. Rev. Lett. 97 (2006) 266405.

[38] C. Gomez-Navarro, M. Burghard, and K. Kern, Nano Lett. 8 (2008) 2045. 